\title{
Study on AC Resistance of Layering Enamelled Stranded Conductor
}

\author{
Zhenxing Jiang ${ }^{1}$, Liming Yang ${ }^{1}$, Zhien $_{\text {Zhu }}{ }^{1}$ \\ ${ }^{1}$ NARI Group Corporation(State Grid Electric Power Research Institute), 211000, Nanjing Jiangsu, China
}

\begin{abstract}
In recent years, with the rapid development of large-length ultra-high-pressure voltage extrusion insulated submarine cable technology, the cable system in the medium-length offshore power grid interconnection project has been gradually replaced by XLPE insulation by traditional oil-paper insulation. There are many large cross-section conductors used in long-distance power transmission cables, and according to the IEC 60287-1-1, it is recommended to use Milliken conductors to reduce the AC resistance of conductors, at the same time, in submarine cables, there is a high requirement on the water-blocking performance of conductors and the water resistance of Milliken conductors is poor, so it is not used in submarine cable conductors. This paper puts forward a layering enamelled stranded conductor and a calculation method of AC / DC resistance ratio for it. The validity of the calculation method is verified by experimental tests. At the same time, the calculation shows that the AC / DC resistance of the conductor at $1800 \mathrm{~mm}^{2}$ nominal cross section can be reduced by $16 \%$ compared with the conventional round compacted conductor in the prior art. The effectiveness of this conductor in reducing AC resistance is verified by finite element simulation.
\end{abstract}

\section{Introduction}

The engineering application of large-length ultra-high voltage cross-linked polyethylene insulated (XLPE) submarine cable technology can not only eliminate the marine leakage of oil-filled cables and the protecting forests anxiety, but also has the advantages of environmental protection, high reliability and low cost.

Some experts and scholars at home and abroad have studied the AC resistance characteristics of conductors with different structures. Many scholars use complicated models and complicated electromagnetic field formulas to calculate the AC resistance of segmented cable conductors, as in K. Sugiyama's literature. In the latest edition of IEC 60287[1], the recommended values of the skin effect correction factor $k_{s}$ for different conductors such as solid round copper conductors are given. The file 272 of CIGRE B1.03[2] working group gives a detailed introduction about the theoretical calculation, finite element simulation and actual measurement of $\mathrm{k}_{\mathrm{s}}$ for the split conductors with various monofilament surface states. However, as of now, there is no literature to report the calculation method of AC resistance and the distribution of conductor currents of insulated stranded round conductors with layered enameled wires. This paper presents a new low AC resistance conductor that is compatible with the traditional cable insulation structure and has the same appearance. The AC resistance of the conductor is calculated and the actual resistance test is performed. The current distribution under the alternating electric field of the conductor is analyzed though finite element simulation.

\section{AC Resistance Calculation}

In this paper, two different conductors are theoretically calculated and tested, and the current distribution is simulated. These two kinds of conductors are copper wire strands conductor(CWSC) and (layered enamelled stranded conductor (LESC)). Both conductors have a cross-sectional area of 1800 square millimetre, with outer diameter 51 millimeter, compression factor 0.93 .

\subsection{Copper Wire Stranded Conductor ( CWSC )}

The increase in resistance caused by AC resistance compared to DC resistance skin effect is the main reason. In this paper, the calculation of AC resistance ignores the proximity effect. In the calculation of AC resistance of conductors in IEC 60287-1-1, a simplified formula for calculating AC resistance of round copper stranded conductors based on a theoretical model is provided as shown in equation (1).

$$
R=R^{\prime} \cdot\left(1+y_{s}+y_{p}\right)
$$

Since the relationship between the distance $S$ between the axial lines of the three single-core submarine cables and the submarine cable outer diameter $D$ satisfies $S \geqslant 5 D$, the proximity effect coefficient, $y_{p}=0$, can be ignored, as shown in equation (2).

$$
R=R^{\prime} \cdot\left(1+y_{s}\right)
$$

The skin effect factor $y_{s}$ formula is equation (3): 


$$
y_{s}=\frac{x_{s}{ }^{4}}{0.8 x_{s}{ }^{4}+192}
$$

The $x_{s}$ in the equation: intermediate parameter, $x_{s}{ }^{2}$ is equation (4):

$$
x_{s}^{2}=\frac{8 \pi f}{R^{\prime}} \cdot 10^{-7} \cdot k_{s}
$$

$R^{\prime}$ : copper conductor DC resistance at 20 degrees centigrade;

$f$ : operating voltage frequency of conductor;

$k_{s}$ : correction factor for the calculation formula and the actual test value;

According to the above formula, the skin effect factor of common copper stranded conductor can be calculated, and then the AC resistance of conductor can be calculated. The values of $k_{s}$ have been given for conductors of different surface states in the standard IEC 60287-1-1, as shown in table 1.

Table 1. IEC 60287- 1-1 gives $\mathrm{k}_{\mathrm{s}}$ recommendations for different conductors

\begin{tabular}{|c|c|}
\hline $\begin{array}{c}\text { type of the } \\
\text { conductor (copper) }\end{array}$ & ks \\
\hline Round, solid 1 & 1 \\
\hline Round, stranded & 1 \\
\hline $\begin{array}{c}\text { Round, Milliken } \\
\text { (insulated wires) }\end{array}$ & 0.35 \\
\hline $\begin{array}{c}\text { Round, Milliken (bare } \\
\text { uni-directional wires) }\end{array}$ & 0.80 \\
\hline
\end{tabular}

Where in $k_{s}$ calculation value of copper wire strands is 1 , and skin effect factor $\mathrm{y}_{\mathrm{s}}=1.37$ is calculated according to $1800 \mathrm{~mm}^{2}$ copper stranded conductor.

\subsection{Layered Enamelled Wire Stranded Conductor ( LESC )}

The standard IEC 60287-1-1 determines the AC/DC resistance ratio of different structures such as oxidized monofilament and enamelled wire conductor by calculating the value of the correction factor $\mathrm{k}_{\mathrm{s}}$ in the theoretical derivation formula of the skin effect factor $\mathrm{y}_{\mathrm{s}}$. In the 2014 version of the standard, the measured values of the $\mathrm{k}_{\mathrm{s}}$ values of several common cable conductors are given in tabular form.

Some experts and scholars at home and abroad have studied the AC resistance characteristics of conductors with different structures[3][4]. Many scholars use complicated models and complicated electromagnetic field formulas to calculate the AC resistance of segmented cable conductors[5][6][7][8][9], as in $\mathrm{K}$. Sugiyama's literature[10][11].

Working group file 272 of CIGRE B1.03 introduces in detail the theoretical calculation, finite element simulation and actual measurement of $\mathrm{k}_{\mathrm{s}}$ for bare copper monofilaments, enameled monofilaments and segmented conductors with oxidized monofilaments. However, the calculation method of the skin effect factor $y_{s}$ in the case of conductor with partial enamelled wire monofilament and partial copper monofilament is not given and no one else has studied it.

The skin effect factor of mixed stranded conductors with enamelled wires and copper wires is no longer applicable to the above formula in CIGRE file 272, and the correction factor $k_{s}$ in the formula needs to be calculated by a special method.

This paper presents a calculation method of conductor AC resistance based on radial conductance fitting of conductor stranded monofilaments. The method firstly calculates the structural parameters of conductor, and then calculates $S_{r}$. $S_{r}$ is defined as the ratio of the surface radial conductance between the stranded single wires. Using the $S_{r}$ and $k_{s}$ data of the single wire enameled insulated stranded conductor and the copper wire stranded conductor in the file 272 of CIGRE B1.03, the relationship curve between $S_{r}$ and $k_{s}$ is obtained by least squares fitting, and finally the $\mathrm{k}_{\mathrm{s}}$ of the layering enameled stranded conductor is calculated.

Let us make some assumptions before calculating:

1. The film is not damaged during the stranding process of the enameled single wire;

2. The film thickness is negligible compared to the monofilament diameter;

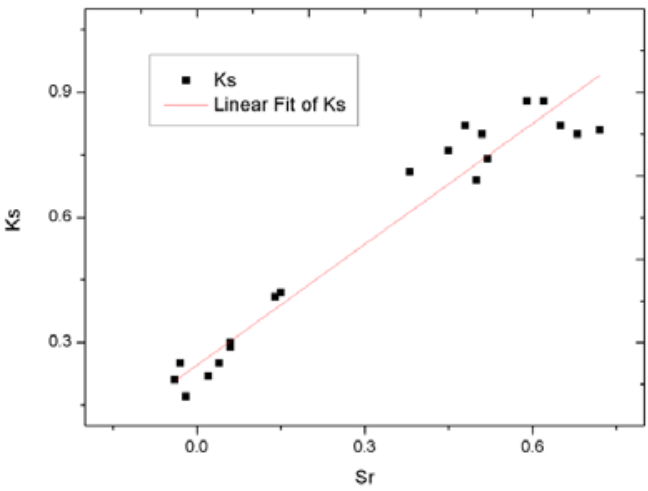

Fig. 1 Fitting curve of $k_{s}$ and $S_{r}$

The data points are calculated by least squares fitting, and the fitting results are shown in Fig. 1. The formula is shown in formula (5).

$$
k_{s}=0.245+0.967 \times S_{r}
$$

For LESC, $S_{\mathrm{r}}$ theoretical formula can be expressed by formula (6):

$$
S_{r}=\frac{\sum_{a=1, b=1}^{c} \gamma_{a b}+\sum_{a=1}^{d} \gamma_{a a}+\sum_{b=1}^{e} \gamma_{b b}}{(n+m) \cdot \gamma_{c u}}
$$

In the formula :

$\alpha$ : correction factor with $\mathrm{S}_{\mathrm{r}}$ measured data;

a : indicates that the monofilament is a bare copper wire;

b: indicates that the monofilament is an enamelled wire;

$\mathrm{c}$ : indicates that one of the adjacent two wires is a bare copper wire, and the other is the combined number of enamelled wire; 
$\mathrm{d}$ : indicates the combined number of adjacent two wires that are bare copper wires;

e : indicates the combined number of two adjacent wires, both of which are enamelled wires;

$\mathrm{n}$ : indicates the number of bare copper wires;

$\mathrm{m}$ : indicates the number of enamelled wires;

$\gamma_{a a}$ : indicates the surface radial conductance when two adjacent wires are both bare copper wires;

$\gamma_{b b}$ : indicates the surface radial conductance when two adjacent wires are both enamelled wires;

$\gamma_{a b}$ : indicates that one of the adjacent two wires is a bare copper wire, and the other is the surface radial conductance of the enamelled wire;

$\gamma_{c u}$ : indicates the stranded single wire conductance of copper conductor monofilaments;

Because the insulation resistance of the enamelled single wire is high, its conductivity is negligible compared to copper conductor, so $\gamma_{a b}=\gamma_{b b}=0$, and known from the layering insulated conductor structure, $\mathrm{d}$ $=\mathrm{n}, \gamma_{a a}=\gamma_{c u}+\gamma_{j c}, \gamma_{j c} \ll \gamma_{c u}$.

At the same time, the method of multiplying the correction factor is used to correct the difference between the theoretical calculation value and the actual measurement value. Based on the actual measurement data in file 272 of CIGRE B1.03, the factor is taken as 0.55 . Thus, the actual $S_{\mathrm{r}}$ calculation formula is shown in equation (7).

$$
\begin{aligned}
& S_{r}=\frac{\alpha \cdot\left(\sum_{a=1, b=1}^{c} \gamma_{a b}+\sum_{a=1}^{d} \gamma_{a a}+\sum_{b=1}^{e} \gamma_{b b}\right)}{(n+m) \cdot \gamma_{c u}} \\
& =\frac{\alpha \cdot n \cdot\left(\gamma_{c u}+\gamma_{j c}\right)}{(n+m) \cdot \gamma_{c u}}=\frac{\alpha \cdot n \cdot \gamma_{c u}}{(n+m) \cdot \gamma_{c u}} \\
& =\frac{\alpha n}{n+m}
\end{aligned}
$$

The structure of the LESC is shown in Fig. 2, the outermost layer in the Fig. 2 is bare copper monofilament, twisted into one layer of enamelled wire and bare copper monofilament in sequence from the inside, and so on.

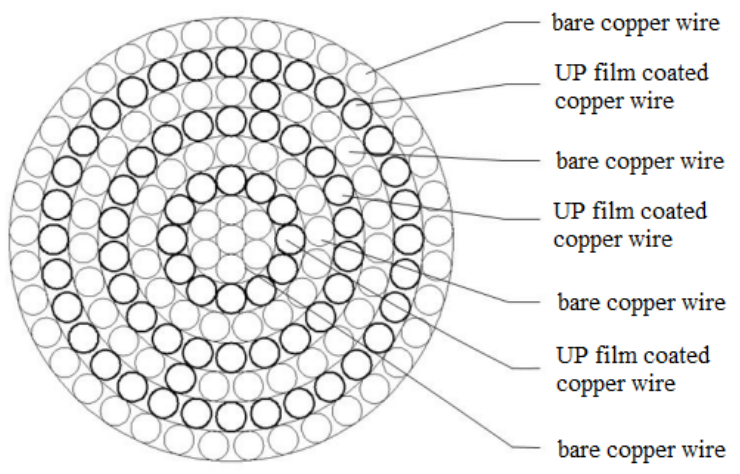

Fig. 2 Structure diagram of LESC

The parameter in Fig. 2 shows as table 2:
Table 2 Structural parameters of layering enamelled stranded conductor

\begin{tabular}{|c|c|c|c|c|}
\hline $\begin{array}{c}\text { copper } \\
\text { wire }\end{array}$ & $\begin{array}{c}\text { enameled } \\
\text { wire }\end{array}$ & diameter & $\begin{array}{c}\text { cross section } \\
\text { before } \\
\text { compression }\end{array}$ & $\begin{array}{c}\text { cross section } \\
\text { after } \\
\text { compression }\end{array}$ \\
\hline 88 & 66 & $4 \mathrm{~mm}$ & $1934 \mathrm{~mm}^{2}$ & $1800 \mathrm{~mm}^{2}$ \\
\hline
\end{tabular}

$\mathrm{S}_{\mathrm{r}}=0.31$ is calculated for the LESC of the structure in figure 1 , and $\mathrm{k}_{\mathrm{s}}=0.55$ and $\mathrm{y}_{\mathrm{s}}=0.14$ are further calculated. Therefore, the AC / DC resistance ratio at 20 degrees centigrade $R_{a c} / R_{d c}=1+\mathrm{y}_{\mathrm{s}}=1.14$ can be calculated.

\section{AC Resistance Test}

In this paper, an $\mathrm{AC}$ resistance tester is used to test the AC resistance on CW and LESC. The test is based on the four-terminal method.The comparison between the AC/DC resistance ratio test results and the theoretical calculation results is shown in Fig. 3.

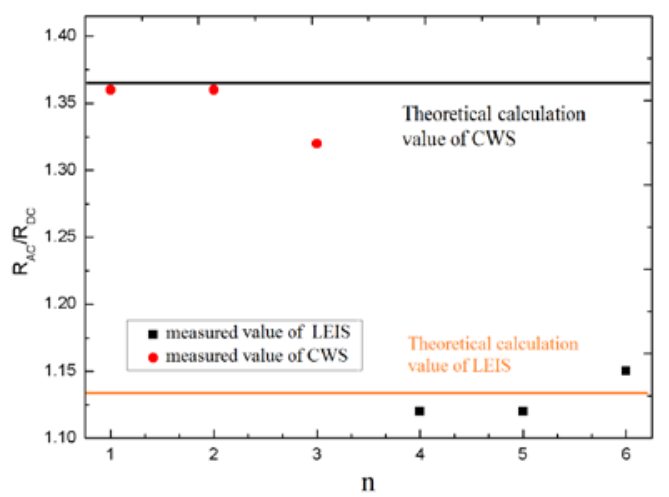

Fig. 3 Comparison between test value and theoretical value

Comparing the theoretical values with the test values, it can be seen that the deviation values of CWSC and LESC are not more than 5 percent, which shows that the calculation method can meet the requirements of field calculation and accuracy in engineering for calculating the AC/DC resistance ratio of LESCs.

\section{Current Distribution Simulation}

\subsection{Finite Element Simulation}

Since the AC resistance data is more abstract, the current distribution in the conductor can be simulated and analyzed by finite element simulation method, which can directly compare the current distribution differences. It is also widely used in the simulation of various physical processes, such as current distribution in conductors, electric field distribution in insulation[12], etc.

\subsection{Model Building}

The finite element simulation software was used to build the model of 1800 square millimetre CWSC and LESC, defined the material properties and physical fields, and apply a magnetic filed simulation module to apply 500 Ampere, 1000 Ampere and 1500 Ampere currents to the 
conductor. Each stranded wire in the CWSC is defined as pure copper, the remaining gaps are defined as air, each stranded wire in a LESC is defined as pure copper, 10 micron paint film is attached to the monofilament, and the remaining gaps are defined as air.

\subsection{Analysis of Simulation Results}
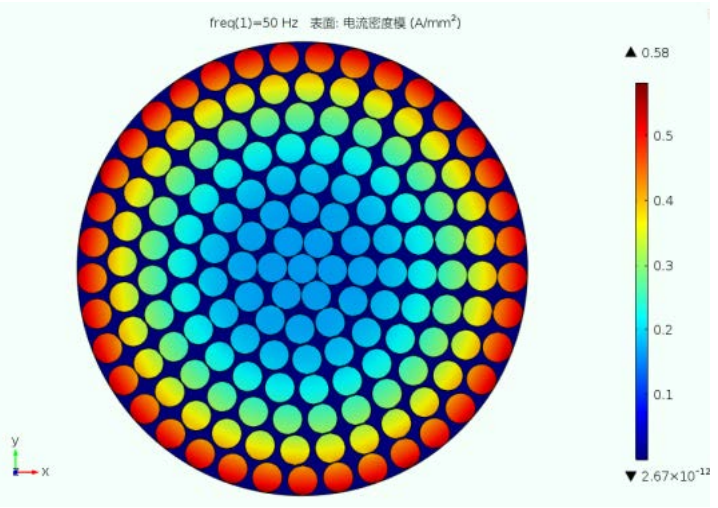

a) $\mathrm{I}=500 \mathrm{~A}, \gamma_{\text {gap }}=10^{-2} \mathrm{~S} / \mathrm{m}$
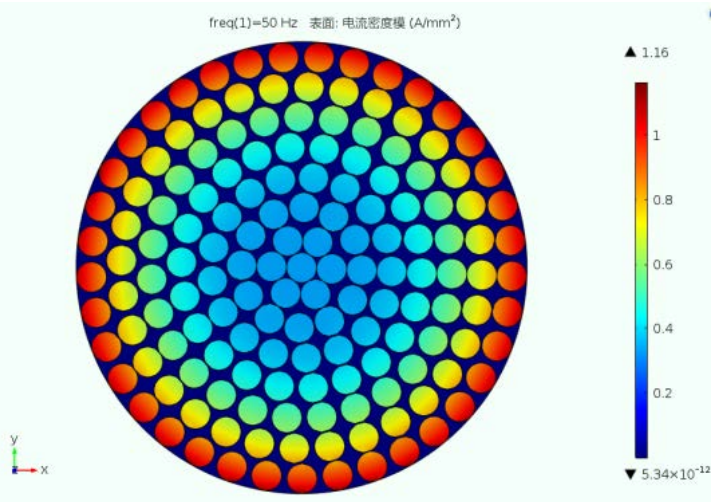

b) $\mathrm{I}=1000 \mathrm{~A}, \gamma$ gap $=10^{-2} \mathrm{~S} / \mathrm{m}$

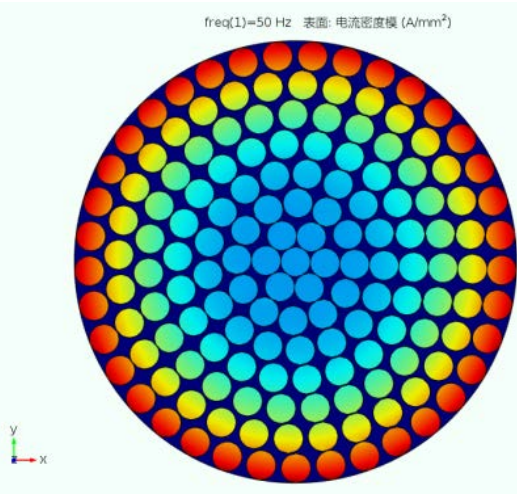

c) $\mathrm{I}=1500 \mathrm{~A}, \gamma$ gap $=10^{-2} \mathrm{~S} / \mathrm{m}$

Fig. 4 current distribution of CWSC

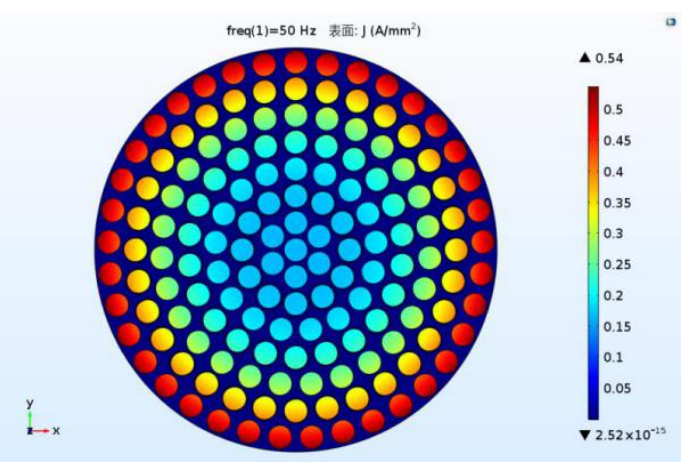

a) $\mathrm{I}=500 \mathrm{~A}, \gamma_{\text {paint }}=9 \times 10^{-7} \mathrm{~S} / \mathrm{m}, \gamma_{\text {gap }}=10 \mathrm{~S} / \mathrm{m}, \Delta_{\text {film }}=10 \mu \mathrm{m}$

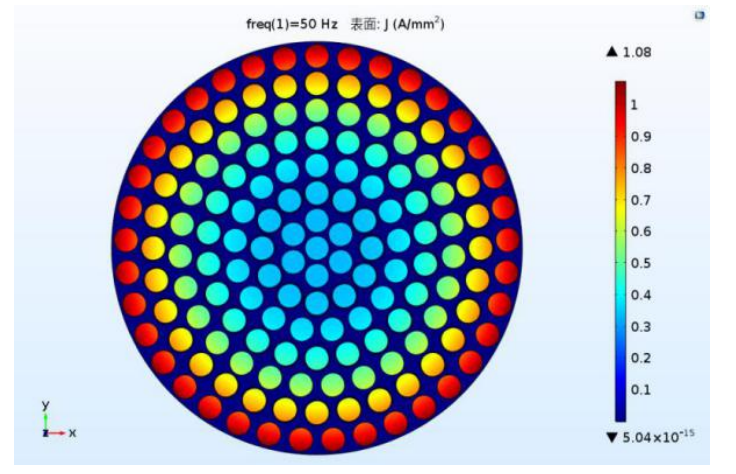

b) $\mathrm{I}=1000 \mathrm{~A}, \gamma_{\text {lacquer }}=9 \times 10^{-7} \mathrm{~S} / \mathrm{m}, \gamma_{\text {gap }}=10 \mathrm{~S} / \mathrm{m}, \Delta_{\text {film }}=10 \mu \mathrm{m}$

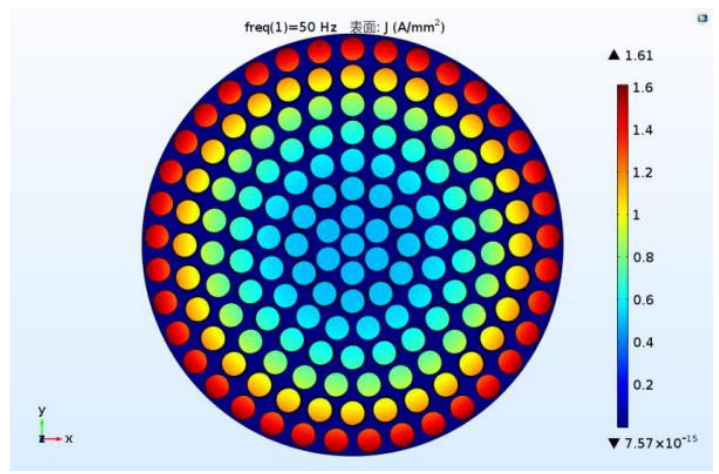

c) $\mathrm{I}=1500 \mathrm{~A}, \gamma$ lacquer $=9 \times 10^{-7} \mathrm{~S} / \mathrm{m}, \gamma$ gap $=10 \mathrm{~S} / \mathrm{m}, \Delta_{\text {film }}=10$ $\mu \mathrm{m}$

Fig. 5 current distribution of LESC

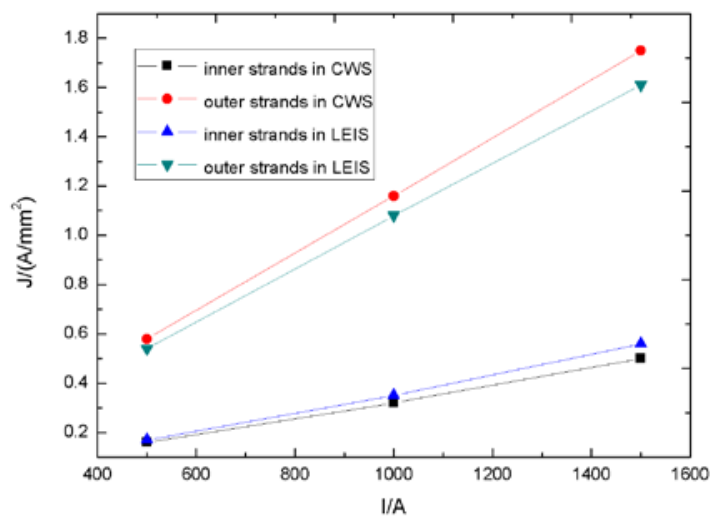

Fig. 6 Results comparison of CWSC and LESC conductor current density 
It can be seen from Fig. 4 and Fig. 5 that the current distribution of the layered enamelled insulated strands is more uniform, and from Fig. 6, the current density of the inner conductor is higher, and the current density of the outer conductor is lower, thus verifying the effect of lowering the AC resistance of the LESC.

The above phenomenon takes advantage of the exponential decay principle of electromagnetic signals propagating from the conductor surface to the interior, that is, skin effect. In engineering, the penetration depth is used to represent the skin effect of the field in good conductors. At the frequency of $50 \mathrm{~Hz}$, the penetration depth of copper is 9.4 millimetre. Formula (8) shows the penetration depth.

$$
d=\sqrt{\frac{1}{\pi f \mu \gamma}}
$$

In the formula :

d: penetration depth;

$\pi$ : circumference rate;

f: electrical signal frequency;

$\mu$ : conductor permeability;

$\gamma$ : conductivities of conductor

It can be seen from the formula that two methods can be taken to reduce the effect of the skin effect, one of which is to reduce the diameter of the monofilament and the other is to increase the surface area of the conductor when the area is fixed.

The technical solution adopted by LESC is to increase the effective surface area of the conductor.

\section{Conclusion}

1. The 1800 square millimetre LESC designed in this paper has the obvious effect of reducing the AC resistance, and the AC/DC resistance ratio is reduced by 16 percent compared with the common conductor.

2. Through AC resistance calculation and actual test, it is found that the calculated values of LESC are consistent with the measured values, and the deviation is small;

3. Finite element simulation shows that the current density of the outer monofilament of the conductor is reduced and the current density of the inner monofilament is increased by adopting LESC, thus the AC resistance of the conductor is finally reduced.

\section{Acknowledgement}

The research work of this paper is funded by the State Grid Corporation science and technology project (5211DS17002F) and is strongly supported by the research team of Professor Wu Weining of NARI Technology Co., Ltd., and thanks for the conductor samples provided by NARI Yinlong Cable Co., Ltd and so on. I hereby express my deep appreciation.

\section{Reference}

1. Cei, Iec /. "IEC 60287-1-1 AMD 1 (2014-11)" (2014).

2. CIGRE WORKING GROUP B1.03 272 Large Cross-sections and composite screens design (2005).

3. E. H. Salter, "Problems in the Measurement of A-C Resistance and Reactance of Large Conductors". American Institute of Electrical Engineers, Transactions of the 67.2 .Jan, 1390-1397 (1948).

4. Udo Fromm, "Optimized conductors for XLPE cables with a large cross-section", Euro. Trans. Electr. Power 15.2 Mar. 109-121 (2005).

5. P. Argaut and J.Y. Daurelle, "Calculation method of power cables AC resistance with individually insulated strands", Proc. of Jicable' 99. Bd. 2. Versailles, France, Jun., 582-591 (1999).

6. V.T. Morgan, R.D. Findlay and S. Derrah, "New formula to calculate the skin effect in isolated tubular conductors at low frequencies", Science, Measurement and Technology, IEE Proceedings 147.4 . Jul, 169-171 (2000).

7. C. Katz, G. S. Eager et. al., "Progress in the Determination of AC/DC Resistance Ratios of PipeType Cable Systems", Power Apparatus and Systems, IEEE Transactions on PAS-97.6, 2262-2271 (1978).

8. Dai D, Zhang $X$, Wang J. "Calculation of AC Resistance for Stranded Single-Core Power Cable Conductors" . IEEE Transactions on Magnetics, 50(11):1-4 (2014).

9. SCHRÖDER, Gero, et al. "AC resistance measurements on skin-effect reduced large conductor power cables with standard equipment." 9th International Conference on Insulated Power CablesJicable (2015).

10. K. Sugiyama u. a. "The skin effect of large size conductors for power cables", Conf. IEEJ Tokyo Branch. 6 (1977).

11. K. Sugiyama. "Development of inter-layer insulated segmental conductor with low skin effect for power cables". Elect. Eng. Jpn. 101.6, 98-108 (1981).

12. Riba J R. "Calculation of the ac to dc resistance ratio of conductive nonmagnetic straight conductors by applying FEM simulations" . European Journal of Physics, 36(5):973-984 (2015). 